\title{
CLOUD MOTION ESTIMATION IN SEVIRI IMAGE SEQUENCES
}

\author{
Javier Marcello ${ }^{1}$,Francisco Eugenio ${ }^{1}$, Ferran Marqués ${ }^{2}$ \\ ${ }^{1}$ Signals and Communications Department. University of Las Palmas of Gran Canaria \\ E-mail: fmarcello@dsc.ulpgc.es, feugenio@dsc.ulpgc.es \\ ${ }^{2}$ Signal Theory and Communications Department. Technical University of Catalonia \\ E-mail: ferran@gps.tsc.upc.edu
}

\begin{abstract}
Determination of atmospheric dynamic characteristics from remote sensing imagery is fundamental in weather and climate studies. The SEVIRI radiometer, on board the MSG, with its 12 bands and 15 minutes sensing capability provides an important amount of information for cloud tracking. In this work, we have first conducted a detailed evaluation of twelve region matching techniques in order to select those providing the best results. For this performance evaluation, databases of synthetic and real sequences have been used. Next, the best metrics have been incorporated in a new methodology that includes a preliminary stage that segments cloudy structures to initialize the optimum motion estimation parameters (template size and search window dimensions). Also a study region mask is generated to disable the application of the motion estimation algorithm in unreliable areas, thus, eliminating erroneous vectors and decreasing the computation times.
\end{abstract}

Index Terms - Motion estimation, region matching, clouds, SEVIRI, segmentation.

\section{INTRODUCTION}

The most important instrument installed on board the MSG (METEOSAT Second Generation) is the SEVIRI radiometer (Spinning Enhanced Visible and Infrared Imager), which provides images of the fourth part of the disc and displays terrestrial cloud formations, areas of fog, takes measures of temperature, ozone and other studies about the weather conditions. It has 12 spectral channels and provides 20 times more data compared to its predecessor. Images are generated every 15 minutes, doubling the temporal resolution with respect to the previous METEOSAT. The spatial resolution is $3 \mathrm{~km}$ at nadir, except for the high-resolution channel (HRV) which is $1 \mathrm{~km}$. In addition to the 12 bands, other processed products can be used to track clouds in image sequences. The most important ones are the cloud mask (CLM), CTH (Cloud Top Height) or CLAI (Cloud Image Analysis).
Figure 1 shows an example CTH and CLAI image for May $4^{\text {th }}, 2007$.

On the other hand, determining the apparent motion from time-varying image sequences is a challenging issue in many image processing applications (i.e. medical imaging, video coding, object tracking, assisting in autonomous navigation, modeling the evolution of clouds, ice or oceanic features, etc.).

In the last decades, different strategies have been developed for the estimation of the optical flow, nevertheless, there does not exist yet a robust methodology providing optimum results when applied to the problem of tracking cloudy zones in remote sensing sequences, mainly, due to: (i) the presence of uniform areas without structures, (ii) the different motions appearing at different heights, (iii) the high deformation of the cloudy structures, which can split or merge, (iv) the image degradation due to atmospheric noise, and (v) the fact that gradients are weak and fuzzy, generating several unconnected border lines and making it difficult to identify structures.

Besides that, an in-deep quantitative survey of motion estimation techniques has not yet been carried out. Much of the image motion literature presents examples for a few preferred image sequences which can only be mainly judged qualitatively. As a consequence, a detailed assessment of the performance of those techniques in the remote sensing scenario is essential to select those algorithms providing the most accurate flow fields.

\section{METHODOLOGY}

\subsection{Motion estimation techniques}

Region matching techniques have been the most popular approaches to estimate motion. Though the MCC (Maximum Cross-Correlation) metric is almost exclusively used (see figure 1) in flow estimation by a majority of authors [1]-[5], a number of other metrics exists.

The three basic metrics [6] are: (i) the sum of absolute differences (SAD), (ii) the sum of square differences (SSD), and (iii) the cross-correlation (CC), while the rest are 


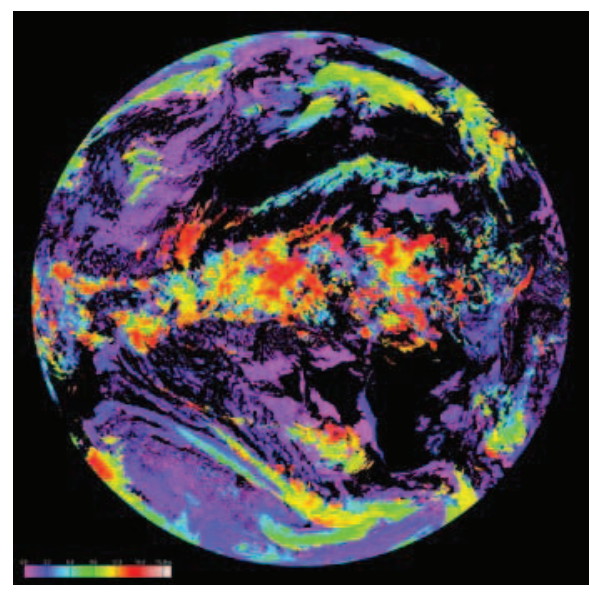

(a)

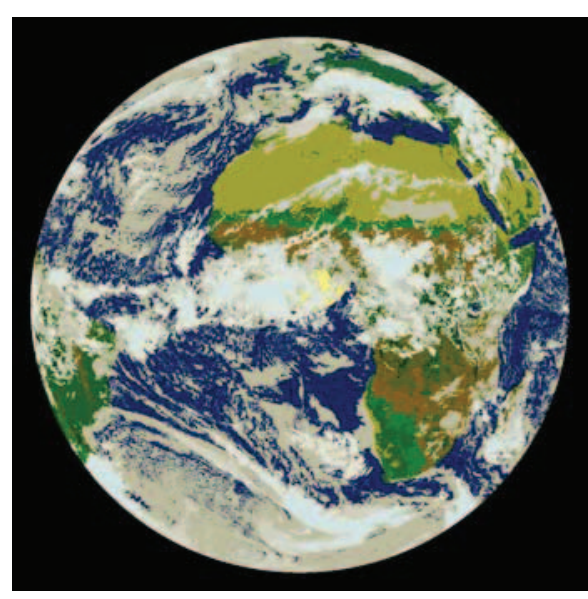

(b)

Figure 1. SEVIRI processed images of for May $4^{\text {th }}, 2007$ : (a) CTH and (b) CLAI.

derived by combinations of normalization and zeromeaning. In these cases the closest match is given by the minima of the SAD, SSD and variants, or by the maxima of the $\mathrm{CC}$ and its variants. In this work, a total of 12 metrics have been implemented: SAD, NSAD (normalized sum of absolute differences), ZSAD (zero-mean sum of absolute differences), NZSAD (normalized zero-mean sum of absolute differences), SSD, NSSD (normalized sum of square differences), ZSSD (zero-mean sum of square differences), NZSSD (normalized zero-mean sum of square differences), CC, NCC (normalized cross-correlation), CCCoef (cross-correlation coefficient), and NCCCoef (normalized cross-correlation coefficient), usually applied under the name of MCC.

The formulations of metrics providing the best results are,

$\operatorname{ZSAD}\left(x, y, d_{x}, d_{y}\right)=\sum_{j=-N}^{N} \sum_{i=-N}^{N}\left|\left[I_{1}(x+i, y+j)-\bar{I}_{1}\right]-\left[I_{2}\left(x+d_{x}+i, y+d_{y}+j\right)-\bar{I}_{2}\right]\right|$

$$
\begin{gathered}
\operatorname{ZSSD}\left(x, y, d_{x}, d_{y}\right)=\sum_{j=-N}^{N} \sum_{i=-N}^{N}\left[\left[I_{1}(x+i, y+j)-\bar{I}_{1}\right]-\left[I_{2}\left(x+d_{x}+i, y+d_{y}+j\right)-\bar{I}_{2}\right]\right]^{2} \\
N Z S S D\left(x, y, d_{x}, d_{y}\right)=\frac{\sum_{j=-N}^{N} \sum_{i=-N}^{N}\left[\left[I_{1}(x+i, y+j)-\bar{I}_{1}\right]-\left[I_{2}\left(x+d_{x}+i, y+d_{y}+j\right)-\bar{I}_{2}\right]\right]^{2}}{\sqrt{\sum_{j=-N}^{N} \sum_{i=-N}^{N}\left[I_{1}(x+i, y+j)-\bar{I}_{1}\right]^{2} \cdot \sum_{j=-N}^{N} \sum_{i=-N}^{N}\left[I_{2}\left(x+d_{x}+i, y+d_{y}+j\right)-\bar{I}_{2}\right]^{2}}} \\
N C C\left(x, y, d_{x}, d_{y}\right)=\frac{\sum_{j=-N}^{N} \sum_{i=-N}^{N} I_{1}(x+i, y+j) \cdot I_{2}\left(x+d_{x}+i, y+d_{y}+j\right)}{\sqrt{\sum_{j=-N}^{N} \sum_{i=-N}^{N}\left[I_{1}(x+i, y+j)\right]^{2} \cdot \sum_{j=-N}^{N} \sum_{i=-N}^{N}\left[I_{2}\left(x+d_{x}+i, y+d_{y}+j\right)\right]^{2}}} \\
\text { NCCCoef }=\frac{\underbrace{N}_{j=-N} \sum_{i=-N}^{N}\left[I_{1}(x+i, y+j)-\bar{I}_{1}\right] \cdot\left[I_{2}\left(x+d_{x}+i, y+d_{y}+j\right)-\bar{I}_{2}\right]}{\sqrt{\sum_{j=-N}^{N} \sum_{i=-N}^{N}\left[I_{1}(x+i, y+j)-\bar{I}_{1}\right]^{2} \sum_{j=-N}^{N} \sum_{i=-N}^{N}\left[I_{2}\left(x+d_{x}+i, y+d_{y}+j\right)-\bar{I}_{2}\right]^{2}}}
\end{gathered}
$$

where $[2 N+1] \mathrm{x}[2 N+1]$ is the size of the template window, $(d x, d y)$ represents the displacement, $\bar{I}$ is the mean value of image $I$ inside the window, and the subscripts 1 and 2 of $I$ indicate the first or second image of the sequence, respectively. It is worth pointing out that, to achieve good motion vectors, special care is needed to select the required template and search window size. Another parameter is the amount of template overlap, which determines the template shift over the image and, as a consequence, the density of vectors recovered.

\subsection{Assessment of techniques}

We have conducted a detailed study of these region matching techniques, using two databases: synthetic and real sequences.

\subsubsection{Synthetic sequences}

The main advantage of synthetic fields is that the groundtruth is available and therefore motion can be tested in a methodical fashion. The synthetic flow fields generated to compare the different algorithms are global translation, rotation, scaling, and affine transformation. These fields are used to warp the first image creating a second synthetic image. Simulations have been performed taking into account typical motion magnitudes and directions.

To achieve more realistic error estimation, after the geometric operation, intensity transformation was also included and the resulting image was degraded with additive Gaussian noise and salt and pepper noise. So, the second image in the sequence is obtained by:

$$
g(x, y)=I T[G T(f(x, y))]+\eta_{G}(x, y)+\eta_{S P}(x, y)
$$


where $g(x, y)$ is the image created from the initial image $f(x, y)$ after the geometric transformation $(G T)$, intensity transformation (IT), and the inclusion of Gaussian noise $\eta_{G}(x, y)$ and salt and pepper noise $\eta_{S P}(x, y)$.

For synthetic sequences a reference flow is available, so the error analysis can be performed through comparison with the recovered field. The parameters we used in this case are the mean and standard deviation of the error in magnitude and angle.

\subsubsection{Real sequences}

When ground-truth is not available, we can still perform error analysis. Quality indicators can be based on the visual analysis of the coherence (in magnitude and angle) in the neighborhood of each vector to detect those clearly erroneous or, alternatively, to visually identify the displacements between features in both images. Finally, the atmospheric knowledge of the area under study provides a number of evidences to assess the correctness of the motion fields. We have selected 50 real sequences using images from the 12 available channels of the SEVIRI sensor on board the MSG satellite.

\subsection{Methodology for cloud motion estimation}

In addition to the quantitative assessment and in order to improve the efficiency of the motion estimation procedure, a new methodology has been developed based on a preliminary stage that segments the cloudy structures to initialize the optimum motion estimation parameters [7]:

(i) The adequate template window size is retrieved from the cloud mask after analyzing the size of the cloudy structures to be able to estimate.

(ii) The search window is related to the maximum displacement between features in the consecutive images of the sequence. So, the image difference of cloud masks allows the determination of the maximum motion within the image for that sequence.

(iii) A study region mask is also generated around gradient areas and disables the calculation of motion vectors in unwanted and uniform zones where a reliable template matching is not possible (Figure 2).

Likewise, a post-processing stage has been included to eliminate unreliable vectors according to magnitude and angular considerations, based on neighbourhood coherence.

\section{RESULTS}

After the extensive study of 12 region-based matching metrics to precisely assess their performance, using databases with 20 synthetic sequences and 50 real sequences, the main results were as follows:

- In synthetic sequences, region matching techniques are robust with regard to intensity variations or spatial

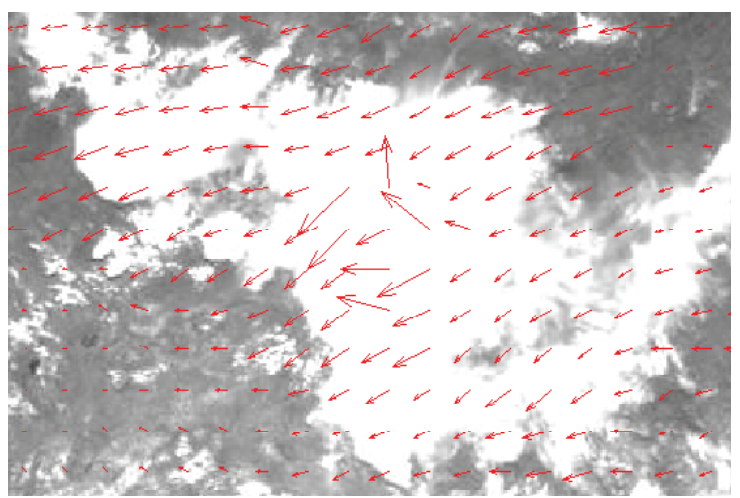

Figure 2. Motion vectors using $\mathrm{MCC}$ for a sequence of visible images of May, 4th 2007 (11:45 and 12:15 am).

displacements within the image. In particular, the best performances were achieved by the ZSAD, ZSSD, NZSSD, and NCC metrics.

- In real sequences, region matching techniques can perfectly estimate the cloud motion (figure 3) and, again, the best results are provided by the previous metrics.

- The most prevalent method (MCC or NCCCoef metric) provides satisfactory results but with slightly larger errors when compared with the other metrics across the two databases.

- As expected, errors increase with time separation between the images of the sequence. However, acceptable results have been achieved for separations of 45 minutes.

- CTH images have been successfully used to group flow vectors according to its height.

- In general terms, motion fields retrieved are similar for all the bands. Greater differences between vectors are found when comparing results for different regions of the spectrum (visible, water vapour, thermal infrared (IR), ozone and carbon dioxide). Figure 4 shows a composition of the fields recovered for all the SEVIRI bands.

- The new methodology improves the overall performance and decreases computation times (the time saved depends on the amount of clouds and their area).

\section{ACKNOWLEDGEMENTS}

This work has been supported by the Universidad de Las Palmas de Gran Canaria (ULPGC07-019 project). We also would like to express our gratitude to EUMETSAT for the access to the HRIT MSG data in the frame of our license agreement for educational use/research project.

\section{REFERENCES}

[1] J.A. Leese, and C.S. Novak, C.S., "An Automated Technique for Obtaining Cloud Motion from Geosynchronous Satellite Data Using Cross Correlation," Journal of Applied Meteorology, v. 10, $\mathrm{n}^{\mathrm{o}} 1$, pp. 118-132, 1971 


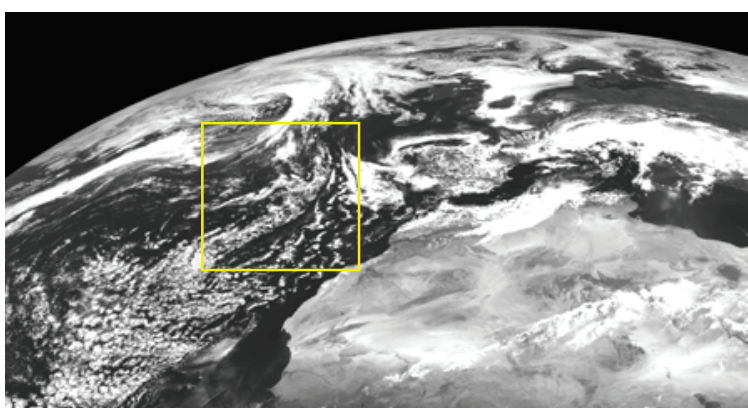

(a)

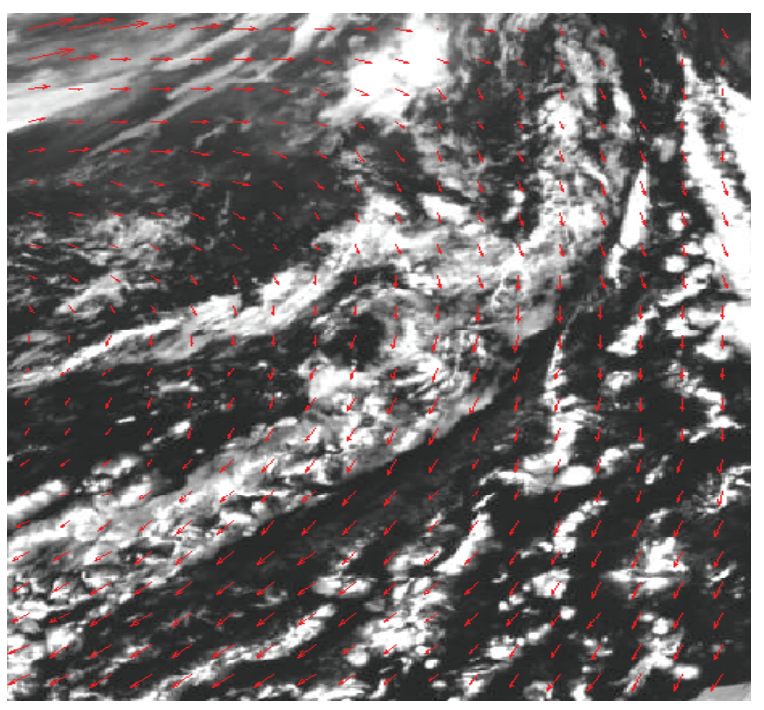

(b)

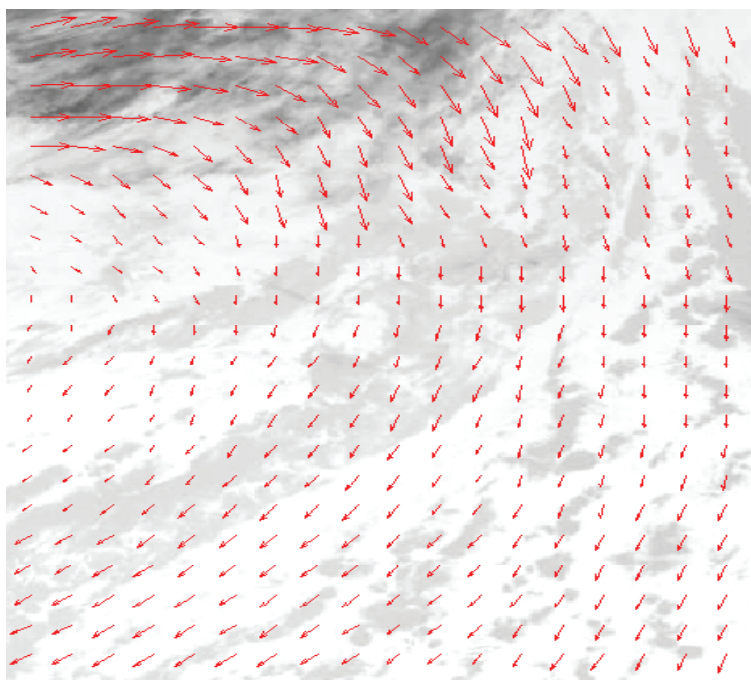

(c)

Figure 3. Estimated motion vectors using ZSAD for a sequence of May, 4th 2007 (11:45 and 12:15 am): (a) Area of interest, (b) motion flow in the visible channel and (c) motion in the thermal IR channel.

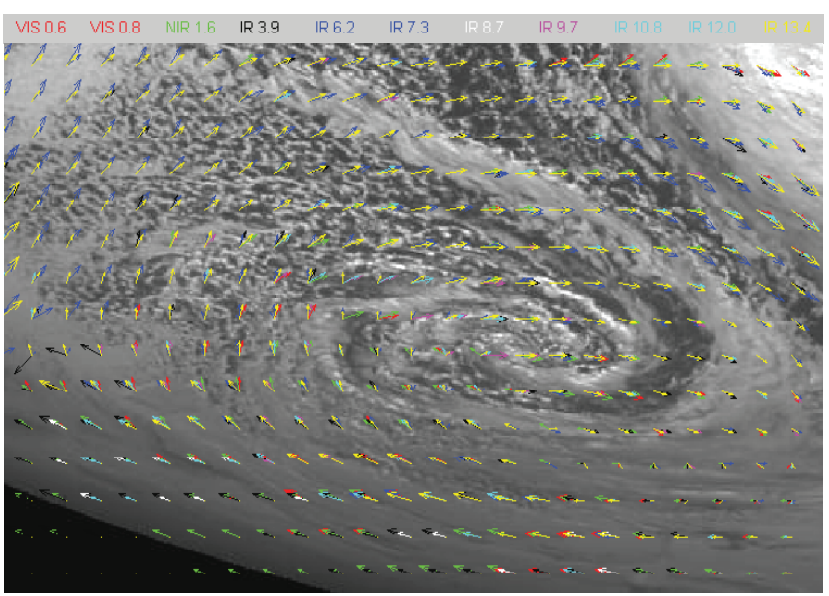

Figure 4. Motion vectors retrieved for each band using the NZSSD metric.

[2] E. Smith and D.R. Phillips, "Automated Cloud Tracking Using Precisely Aligned Digital ATS Pictures," IEEE Transactions on computers, v. 21, nº , pp. 715-729, 1972.

[3] J. Schmetz et al., "Operational cloud-motion winds from Meteosat infrared images," Journal of Applied Meteorology, v. 132 , no. 7, pp. 1206-1225, 1993.

[4] A.N. Evans, "Cloud Tracking Using Ordinal Measures and Relaxation Labelling," IEEE International Geoscience and Remote Sensing Symposium, v. 2, pp. 1259-1261, 1999.

[5] A.N. Evans, "Cloud motion analysis using multichannel correlation-relaxation labelling." IEEE Geoscience and Remote Sensing Letters, v. 3, no. 3, pp. 392-396, 2006.

[6] Andrews, R.J., "Early investigations in optical flow from colour images," Phd. Thesis, Queensland University, Australia, 2003.

[7] J. Marcello, F. Marques and F. Eugenio, "Automatic tool for the precise detection of upwelling and filaments in remote sensing imagery," IEEE Transactions on Geoscience and Remote Sensing, v. 43, pp.1605-1616, 2005. 